\title{
Benefits from aerobic exercise in patients with major depression: a pilot study
}

\author{
F Dimeo, M Bauer, I Varahram, G Proest, U Halter
}

\begin{abstract}
Background-Several reports indicate that physical activity can reduce the severity of symptoms in depressed patients. Some data suggest that even a single exercise bout may result in a substantial mood improvement.

Objective-To evaluate the short term effects of a training programme on patients with moderate to severe major depression.

Methods-Twelve patients (mean (SD) age 49 (10) years; five men, seven women) with a major depressive episode according to the Diagnostic and Statistical Manual of the American Society of Psychiatry (DSM IV) criteria participated. The mean (SD) duration of the depressive episode was 35 (21) weeks (range 12-96). Training consisted of walking on a treadmill following an interval training pattern and was carried out for $\mathbf{3 0}$ minutes a day for $\mathbf{1 0}$ days.

Results-At the end of the training programme, there was a clinically relevant and statistically significant reduction in depression scores (Hamilton Rating Scale for Depression: before, 19.5 (3.3); after, 13 (5.5); $\mathbf{p}=\mathbf{0 . 0 0 2}$. Self assessed intensity of symptoms: before, 23.2 (7); after, 17.7 (8.1); $p=0.006$. Values are mean (SD)). Subjective and objective changes in depression scores correlated strongly $(r=$ $0.66, p=0.01$ ).
\end{abstract}

Conclusions-Aerobic exercise can produce substantial improvement in mood in patients with major depressive disorders in a short time.

(Br f Sports Med 2001;35:114-117)

Keywords: affective disorders; depression; major depression; refractory depression; exercise

Department of Sports Medicine, Freie Universitaet, Berlin, Germany

F Dimeo

I Varahram

Department of

Psychiatry

M Bauer

G Proest

U Halter

Correspondence to:

Dr Dimeo, Freie Universitaet Berlin, Benjamin Franklin

Medical Center, Department of Sports Medicine, Clayallee 229, 14195 Berlin, Germany ferdimeo@zedat.fu-berlin.de

Accepted 14 November 2000 reports on the effects of exercise on depression is the lack of information about the psychiatric diagnosis of participants according to standard references-for example, the Diagnostic and Statistic Manual of the American Society of Psychiatry (DSM IV). Furthermore, most reports provide inadequate information about the characteristics of the sample. For example, two studies enrolled university students with elevated scores on depression scales such as the Beck Depression Inventory; however, participants were not defined as being clinically depressed. $^{34}$ In some studies, exercise programmes were started or carried out at the same time as pharmacological treatment or psychotherapy. ${ }^{56}$ Thus, the relative effects of the two interventions on the observed outcomes cannot be differentiated. Finally, the high percentage of drop outs in most studies renders interpretation of their results difficult.

Despite these problems, most of the evidence indicates positive effects of physical activity on mood. Two meta-analyses suggest that exercise may be as effective as psychotherapy ${ }^{7}$ and more effective than other behavioural interventions ${ }^{8}$ for treating depression. A few studies have used true experimental designs to evaluate the effects of exercise on mood. In the first one, 43 female students with elevated scores in the Beck Depression Inventory were randomly assigned to one of three groups (endurance training, relaxation training, or no treatment). After 10 weeks, depression scores improved significantly in the endurance training group but were unchanged in the relaxation and control groups. ${ }^{3}$ In a recent study, ${ }^{9}$ resistance training over 10 weeks was more effective than a placebo activity to reduce depression scores in elderly people with a Beck Depression Inventory Score $>12$. Finally, in a further study, 40 clinically depressed women carried out one of two exercise programmes (running or weight lifting), or were included in a waiting group without training. After eight weeks, depression scores were significantly lower in both intervention groups than in the controls. ${ }^{10}$

This evidence supports the need for further investigation of the effects of physical activity on patients with more severe forms of affective disorders. Indeed, exercise could have several advantages as treatment for depression-for example, low cost, associated positive effects on physical performance and cardiovascular risk, absence of secondary effects, and potential prevention of future episodes. In a previous study on the effect of an exercise programme on the physical performance of patients after bone marrow transplantation, we observed a substantial improvement in the mood of participants after a few days. ${ }^{11}$ Furthermore, a 
Table 1 Baseline data of patients

\begin{tabular}{ll}
\hline Sex & 7 female, 5 male \\
Age (years) & $49(10)(28-64)$ \\
Body mass index & $26.7(5)(20.8-39.2)$ \\
Setting & 7 outpatients, 5 inpatients \\
Hospital stay (days) & $78(48)(14-136)$ \\
Duration of present depressive episode (weeks) & $35(21)$ (range 12 to 96) \\
\hline
\end{tabular}

Values are mean (SD) followed by the range in parentheses.

${ }^{\star}$ Only for inpatients.

meta-analysis has shown that even a single exercise bout can improve mood. ${ }^{7}$ In view of these findings, we carried out a pilot study to evaluate the effects of a short term aerobic training programme on patients with major depression.

\section{Patients and methods}

A successive series of inpatients and outpatients with major depression were considered for participation in the study. All patients had been diagnosed as suffering from major depressive disorder according to DSM IV criteria. Further inclusion criteria were age between 20 and 65 years, no change in treatment (psychotropic medication, psychotherapy or behavioural therapy) in the six weeks preceding the training programme, hospitalization for at least two weeks without improvement (for hospitalized patients only), a score of 15 or more on the Hamilton Rating Scale for Depression (HAMD, 21 item version), absence of associated organic disease or schizophrenic symptoms, and ability to understand written German. The study was approved by the institutional ethics committee.

A total of 12 patients who fulfilled the inclusion criteria were asked to participate (table 1); all patients gave written informed consent and were recruited for the study. The duration of the present depressive episode was 35 (21) weeks (range 12-96). Ten patients suffered from refractory depression, defined as a lack of improvement after treatment with at least two antidepressants with different mechanisms of action at adequate dosage (tricyclic antidepressants $\geqslant 150 \mathrm{mg}$ /day, serotonin reuptake inhibitors $\geqslant 20 \mathrm{mg}$ /day) for at least four weeks during the present episode.

Maximal physical performance was assessed at the beginning and end of the study with a modified Bruce treadmill test under continuous electrocardiogram monitoring. The test was carried out until subjective exhaustion. Heart rate was continuously assessed; blood pressure and lactate concentration in capillary blood were assessed every three minutes. Maximal oxygen uptake in $\mathrm{ml} / \mathrm{kg} / \mathrm{min}$, an indicator of maximal physical performance, was calculated according to the guidelines of the American College of Sports Medicine. ${ }^{12}$

Training consisted of daily walking on a treadmill; it was started on Mondays and carried out for the following 10 days with breaks on Sundays. The programme was designed according to an interval training pattern. Patients carried out five training bouts of three minutes each; the intensity of effort corresponded to a lactate concentration of $3(0.5)$ $\mathrm{mmol} / \mathrm{l}$ in capillary blood. Between training bouts, patients walked at half speed for three minutes to recover. Intensity of effort was evaluated daily using the Borg Rate of Perceived Exertion scale, a visual analogue scale ranging from 6 ("the effort is very light") to 20 ("the effort is very, very hard"). ${ }^{13}$ On this scale, the selected training intensity corresponded to a value of 13-14 ("somewhat hard"). Heart rate was continuously monitored during training. As exercise heart rate decreased due to training adaptation, treadmill elevation was increased to maintain training intensity. Lactate concentration was controlled every fifth training day. During training, patients were continuously supervised by instructed study personnel; interaction was limited to general comments about walking technique and training related bodily sensations such as degree of effort or muscular complaints.

Severity of depression was rated at the beginning and end of the programme by a psychiatrist using the HAMD (21 item version). Patients rated their mood daily using a visual analogue scale, ranging from 0 ("I feel bad") to 10 ("I feel good"), and at the start, on the fifth training day, and at the end of the training programme with the Scale for Self assessment of Depression (D-S), an instrument commonly used for self evaluation of mood in German speaking countries. ${ }^{14}$ According to the usual clinical criteria, the response to therapeutic intervention was defined as a reduction of $50 \%$ or more or a final score of 10 or less on the HAMD.

\section{STATISTICAL ANALYSIS}

Only data obtained before and after the training programme were included in the statistical analysis. Depression scores before and after training were compared by the two tailed Wilcoxon test. Correlation between subjective and objective improvement in depression was evaluated using the Spearman rank correlation test. All statistical analyses were carried out with GraphPad Prism 3.00 for Windows (GraphPad Software, San Diego, California, USA). A value of $\mathrm{p}<0.05$ was considered statistically significant. Data are expressed as mean (SD) with ranges in parentheses.

\section{Results}

All patients completed the programme without interruption, and no training related complications were observed during the study. At the end of the training programme, we observed a substantial improvement in six patients and a slight improvement in two; the severity of symptoms was unchanged in four patients (fig 1). At the final examination, five patients had scores of 10 or less on the HAMD. On the whole, depression scores were significantly reduced at the end of the training programme in both the objective psychiatric evaluation and the subjective assessment of symptoms (HAMD, $p=0.002 ; \mathrm{D}-\mathrm{S}, \mathrm{p}=0.006$; table 2). A reduction on the HAMD correlated significantly with changes in the self assessment scores $(r=0.66, \mathrm{p}=0.01)$. No differences in 

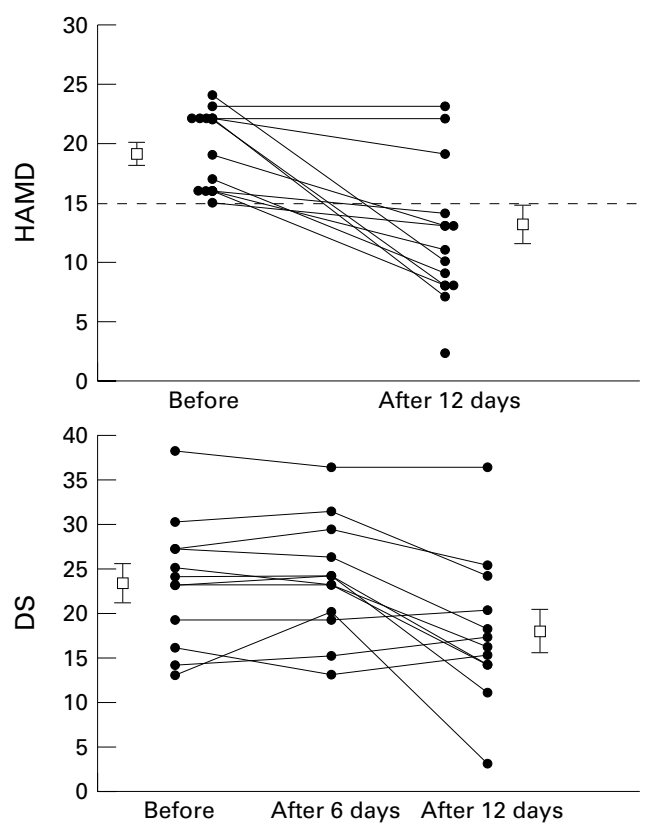

Figure 1 Changes in depression scores during the training programme. Values are expressed as mean (SE). HAMD, Hamilton Rating Scale for Depression; D-S, Scale for Self Assessment of Depression.

the scores of the analogous scale were observed $(\mathrm{p}=0.37)$. Maximal physical performance (expressed as maximal oxygen uptake in $\mathrm{ml} / \mathrm{kg} /$ min) did not change during the study $(\mathrm{p}=1$; table 2). At the end of the study, six patients asked to continue the aerobic training programme.

\section{Discussion}

Our results indicate that, in selected patients with major depression, aerobic training can

Table 2 Changes in depression scores during the training programme

\begin{tabular}{lllll}
\hline & Before & After & CI of the difference & p Value \\
\hline Physical performance (ml/kg/min) & $28.2(10.1)$ & $27.9(10.2)$ & -3.7 to 3.2 & 1.0 \\
Hamilton Rating Scale of Depression & $19.5(3.3)$ & $13.1(5.5)$ & -10.3 to -2.5 & 0.002 \\
Scale for Self Assessment of Depression & $23.2(7)$ & $17.7(8.1)$ & -8.8 to -2.1 & 0.006 \\
Analogue scale & $3.5(2)$ & $4(2.1)$ & -0.8 to 1.8 & 0.42 \\
\hline Values are mean (SD). & & & & \\
CI, Confidence interval. & & & & \\
& & & &
\end{tabular}

Table 3 Individual data of the participants

\begin{tabular}{lllllll}
\hline Patient & Age & Sex & Diagnosis & Setting & Medication & \multirow{2}{*}{ SHAMD } \\
\hline 1 & 59 & $\mathrm{f}$ & 3 & Outpatient & $1,2,4,6$ & -8 \\
2 & 46 & $\mathrm{~m}$ & 2,5 & Outpatient & 1,3 & -5 \\
3 & 36 & $\mathrm{~m}$ & 2 & Outpatient & 3 & 0 \\
4 & 42 & $\mathrm{f}$ & 1,5 & Outpatient & $6,7,4$ & -2 \\
5 & 48 & $\mathrm{f}$ & 2 & Outpatient & 3,6 & -8 \\
6 & 28 & $\mathrm{~m}$ & 2 & Outpatient & $3,4,7$ & -15 \\
7 & 64 & $\mathrm{~m}$ & 2 & Outpatient & 1 & -2 \\
8 & 58 & $\mathrm{~m}$ & 4 & Inpatient & $1,7,8$ & -14 \\
9 & 58 & $\mathrm{f}$ & 4 & Inpatient & 1,2 & -14 \\
10 & 49 & $\mathrm{f}$ & 2 & Inpatient & 1,7 & -6 \\
11 & 56 & $\mathrm{f}$ & 2 & Inpatient & 1,5 & -3 \\
12 & 56 & $\mathrm{f}$ & 4 & Inpatient & 6,7 & 0 \\
\hline
\end{tabular}

Diagnosis: 1, major depressive disorder, single episode, moderate; 2, major depressive disorder, recurrent, moderate; 3, bipolar I disorder, most recent episode depressed, moderate; 4, bipolar I disorder, most recent episode depressed, severe; 5 , dysthymic disorder.

Medication: 1, lithium carbonate; 2, carbamazepine; 3, tricyclic antidepressant; 4, selective serotonin reuptake inhibitor (SSRI); 5, monoaminooxidase inhibitors; 6, L-thyroxine; 7, neuroleptic; 8, sedative.

$\triangle$ HAMD, Reduction in the Hamilton Rating Scale of Depression between both assessments before and at the end of the training programme. produce a substantial improvement in symptoms in a short time. These results are of clinical relevance for obvious reasons. Given the fact that antidepressive drugs have latency times of two to four weeks before any therapeutic effect, the observed outcomes indicate a clinical benefit not obtainable with currently available pharmacological treatments. Furthermore, a substantial percentage of patients show no improvement despite an optimal dosage of antidepressants. For these patients, aerobic training could offer a safe therapeutic option. Indeed, in our study, five of 10 patients with treatment refractory depression showed a substantial improvement in symptoms during the training programme.

In this study, we did not find any differences in the baseline data of responders and nonresponders to exercise (table 3). Previous papers have reported a positive correlation between severity of depressive symptoms and effects of physical activity on mood. ${ }^{8}$ However, the small sample size of our study makes analysis of response patterns difficult.

Most previous publications on the effects of exercise on depression were based on self assessment of patients; only two reports included an objective psychiatric assessment. ${ }^{10}$ In our study, objective changes in severity of disease according to the HAMD showed a high correlation with changes in self assessment of mood; the concordance between the two evaluations adds substantial evidence to the positive effects of aerobic training in this setting.

Several mechanisms can explain the mood improvement in our patients. Exercise produces changes in the concentration of several biologically active molecules such as adrenocorticotrophic hormone, cortisol, catecholamines, opioid peptides, and cytokines, ${ }^{15} 16$ which have been reported to affect mood or are involved in the physiopathology of affective disorders. Moreover, some evidence suggests that exercise can modify the concentration of neuroactive substances in the central nervous system. ${ }^{17}$ However, factors such as motivation, expectancy, and human contact could also influence the mood of participants. However, at the time of participation in the study, most of the patients had been taking part in a programme of therapeutic activities-for example, work or occupational therapy - for several months without any improvement in symptoms. Therefore we feel that psychological or motivational factors alone cannot fully explain the observed outcomes. Further investigations are necessary to determine the mechanisms behind the improvement in mood observed in the study participants.

It is a well known fact that the intensity of symptoms can fluctuate during a depressive episode. As the present investigation did not include a control group, it is difficult to evaluate the effect of spontaneous mood changes in the observed outcomes. However, the magnitude of symptom reduction, the temporal association between the start of the training programme and clinical improvement, and the consistency of mood changes among the 
patients strongly suggest aerobic exercise as a cause of the improvement observed in the study participants. Indeed, five patients (42\%) fulfilled the criteria of clinical response to treatment (defined as a reduction of $50 \%$ or a final score lower than 10 on the HAMD).

We believe that our results are grounds for cautious optimism. In the light of these positive findings, a randomised controlled study has been started to evaluate the effects of an aerobic training programme on patients with major depression.

We thank Sebastian Fetscher, MD, for critically reviewing the manuscript.

1 Folkins $\mathrm{CH}$. Effects of physical training on mood. 7 Clin Psychol 1976;32:385-7.

2 Williams JM, Getty D. Effects of levels of exercise on psychological mood states, physical fitness, and plasma beta-endorphin. Percept Mot Skills 1986;63:1099-105.

3 McCann IL, Holmes DS. Influence of aerobic exercise on depression. F Pers Soc Psychol 1984;46:1142-7.

4 Stein PN, Motta RW. Effects of aerobic and nonaerobic Stein PN, Motta RW. Effects of aerobic and nonaerobic
exercise on depression and self-concept. Percept Mot Skills exercise on dep

5 Martinsen EW, Hoffart A, Solberg O. Comparing aerobic with nonaerobic forms of exercise in the treatment of clinical depression: a randomized trial. Compr Psychiatry 1989 30:324-31.
6 Martinsen EW, Medhus A, Sandvik L. Effects of aerobic exercise on depression: a controlled study. BMF 1985;291: 109 .

7 North TC, McCullagh P, Tran ZV. Effect of exercise on depression. Exerc Sport Sci Rev 1990;18:379-415.

8 Craft LL, Landers DM. The effect of exercise on clinical depression and depression resulting from mental illness: a meta-analysis. fournal of Sport and Exercise Psychogy 1998;20:339-57.

9 Singh NA, Clements KM, Fiatarone MA. A randomized controlled trial of progressive resistance training in depressed elders. F Gerontol A Biol Sci Med Sci. 1997;52: M27-35.

10 Doyne EJ, Ossip-Klein DJ, Bowman ED, et al. Running versus weight lifting in the treatment of depression. 7 Consult Clin Psychol 1987;55:748-54.

11 Dimeo F, Bertz H, Finke J, et al. An aerobic exercise program for patients with haematological malignancies after bone marrow transplantation. Bone Marrow Transplant 1996;18:1157-60.

12 American College of Sports Medicine. Guidelines for exercise testing and prescription. Philadelphia: Lea \& Feibiger, 1995.

13 Borg G. Perceived exertion as an indicator of somatic stress. Scand F Rehabil Med. 1970;3:92-8.

14 Zerssen D, Strian F, Schwarz D. Evaluation of depressive states, especially in longitudinal studies. In: Pichot P, Olivier-Martin R, eds. Psychological measurements in psychopharmacology. Basel: Karger Verlage, 1974:189-202.

15 McArdle WD, Katch FI, Katch VL. Exercise physiology: Baltimore: Williams \& Wilkins, 1996.

16 Verde TJ. Short-term exercise and immune function. In: Watson RR, Eisinger M, eds. Exercise and disease. Boca Raton, FL: CRC Press, 1992:71-88.

17 Chaouloff F. Effects of acute physical exercise on central serotonergic systems. Med Sci Sports Exerc 1997;29:58-62.

Take home message

For selected patients with moderate to severe major depression, aerobic exercise may result in a substantial improvement in symptoms in a short time.

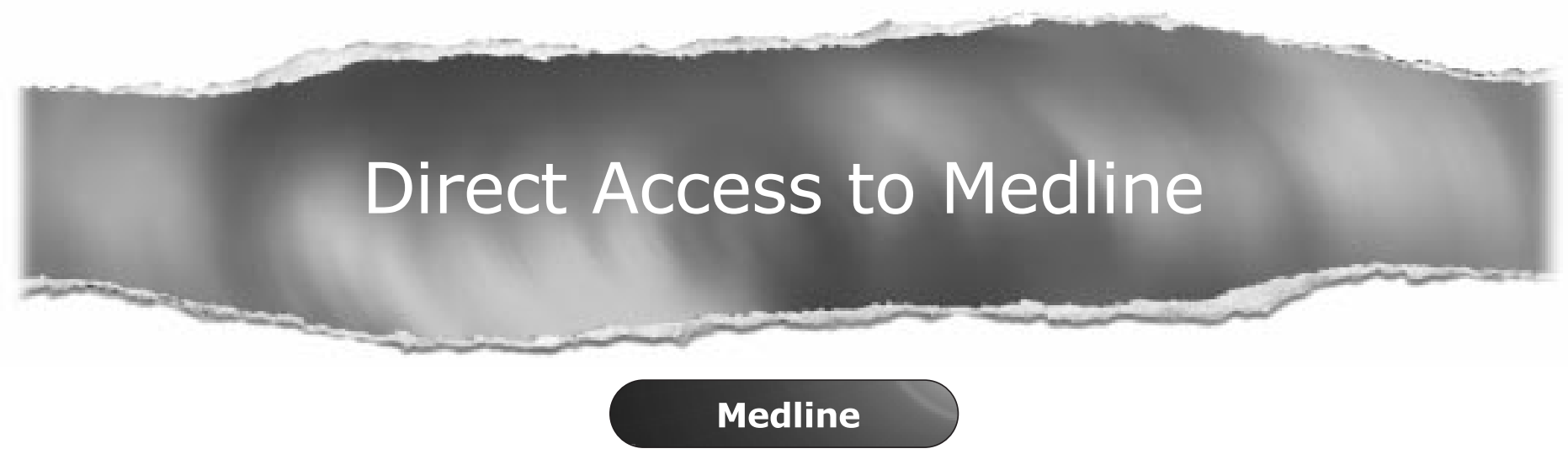

Link to Medline from the homepage and get straight into the National Library of Medicine's premier bibliographic database. Medline allows you to search across 9 million records of bibliographic citations and author abstracts from approximately 3,900 current biomedical journals.

\section{www.bjsportmed.com}

